\title{
Modelling the defrost process in complex geometries - part 2: wall-function based coupling to a multi-region CFD solver
}

\author{
Ellen Hertle ${ }^{1}$, Simon van Buren ${ }^{1}$, Patric Figueiredo ${ }^{1}$, Reinhold Kneer ${ }^{1}$, and Wilko Rohlfs ${ }^{1, *}$ \\ ${ }^{1}$ Institute of Heat and Mass Transfer, RWTH Aachen University, Augustinerbach 6, 52056 Aachen, \\ Germany
}

\begin{abstract}
Presence of frost in air coolers reduces the performance and thus has to be removed frequently. A method commonly applied for frost removal is hot-gas defrosting. For the efficient design of this periodically performed operating sequence, a precise prediction of the process is essential. A CFD simulation is a capable tool for this evaluation. This paper presents an integration of a defrost model into a CFD solver of the software OpenFOAM. The multi-region properties implemented in the existing code are adapted for the metal fin and tube structure and the air flow channel. The extension of the solver for evaluating the frost layer is accomplished by an interfacial wall function which models the frost characteristics and performs the thermal coupling. The extended solver is tested in a simplified environment, focusing on a fin-tube section. The simulated course of the defrost process and the total defrost time are discussed.
\end{abstract}

\section{Introduction}

Frost formation occurs in different technical applications involving cooling of moist air and other gases. In general, frost grows on surfaces for which the temperature is both below the dew point of the surrounding moist air and below the freezing point of water. In most cases frost formation is an undesired phenomenon, creating an isolating layer of ice which negatively affects the performance of heat exchangers. Avoiding frost formation is not always possible, such that defrosting strategies are important for air cooling systems [1]. Relevant aspects of the applied methods are defrosting time, pressure drop across the evaporator, energy consumption, overall efficiency, and defrost cost [2].

The first theoretical model describing the defrost process has been developed by Sanders [3], who divided the entire process into different stages including preheating and melting. Machielsen [1] used the model of Sanders [3] to determine the optimal cooling-defrost cycle. Using a simplified circular geometry, Hoffenbecker [4] investigated the defrost time and required defrost energy in industrial air-cooling evaporators.

A more detailed model was developed by Dopazo [5], dividing the defrost process into six different stages, including preheating, different resistances in water and air layers, and

*Corresponding author: rohlfs@wsa.rwth-aachen.de 
evaporation. The above mentioned models use a strongly simplified geometry, neither accounting for natural convection nor varying air temperature in the evaporator. Further, only the melting process at the wall-frost interface is considered, and not at the opposed airfrost interface. A modelling approach for frost formation in complex geometries was for instance developed by Ellgas [6], coupling a commercial CFD-code with a frost model. The model was applied to study the frosting of an injector nozzle and blocking effects of the fluid region due to frost growth was accounted for. Kim [7] presented a numerical model for predicting frost behaviour on a heat exchanger fin under frosting conditions, taking into account fin heat conduction and three-dimensional air flow. Lenic [8] proposed a transient two-dimensional model of frost formation that includes air and frost subdomains as well as a boundary condition on the air-frost interface. A subsequent study determined the optimal duration of the cooling cycle and the optimal starting point for the defrost process [8].

In our approach, a three-dimensional CFD code is coupled with a multi-stage defrost model. The CFD code considers different solid and fluid regions which are coupled by a wall-function including the defrost model. Thus, spatially and temporally varying temperatures at the frost-wall and the frost-air interface can be analysed throughout the defrost process. With this model, defrosting of air-coolers with complex shapes can be investigated.

\section{Modelling approach}

Fig. 1 shows a wall, partially covered by a frost layer of thickness $\delta_{\mathrm{f}}$, which is situated between the wall and a gaseous atmosphere (air). In the frost region, $\mathrm{T}_{\mathrm{WF}}$ and $\mathrm{T}_{\mathrm{AF}}$ denote the temperatures at the wall-frost and the air-frost interfaces, respectively. Due to the thermal capacity of the frost and the latent heat required for the phase change, heat fluxes at both interfaces are not identical.

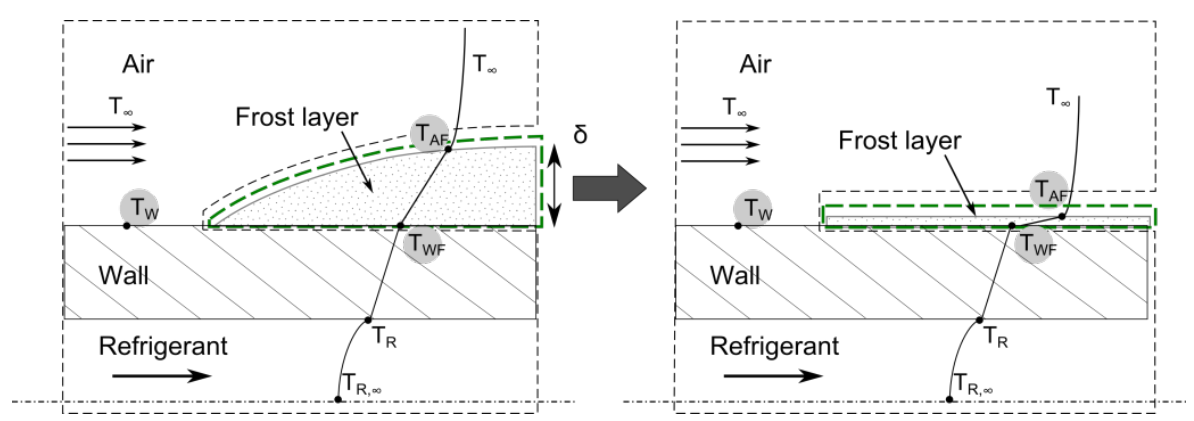

Fig. 1. Modelling approach with an infinitesimal thin frost layer. One-dimensional frost model (left) and zero-dimensional modelling approach (right).

The expansion of the frost layer into the fluid region can influence the flow, due to a partial narrowing of the flow channel. To fully resolve the effects resulting from the frost layer's expansion on the air flow, a computational method including an adaptive grid [6] or an interface tracking method is necessary [9], which require an high computational and modelling effort. Alternatively the expansion of the frost layer in the three-dimensional domain can be reduced to an infinitesimally thin layer between wall and air region (see Fig. 1). This allows a simple description of the frost layer as a wall function in the CFD model, coupling the thermal boundary conditions of the solid-fluid interfaces. Note that this approach neglects the expansion of the frost layer and thus does not account for flow 
channel narrowing or blocking. Nevertheless, the virtual and time-dependent frost layer thickness is integrated in the defrost model and remains a crucial parameter significantly influencing the energy requirement for defrosting.

\subsection{Conservation equations in the solid and fluid regions}

The problem of defrosting in heat exchangers involves solid and fluid regions, for which a multi-region approach is required. In the solid region, the three-dimensional transient equation for energy conservation in terms of enthalpy reads

$$
\frac{\partial\left(\rho_{\mathrm{Wall}} \mathrm{h}\right)}{\partial \mathrm{t}}=\nabla \cdot\left(\alpha_{\mathrm{Wall}} \nabla \mathrm{h}\right)
$$

In the fluid region, the three-dimensional energy conservation equation with the additional terms of convection yields

$$
\frac{\partial\left(\rho_{\text {Air }}\right)}{\partial \mathrm{t}}+\nabla \cdot\left(\rho_{\text {Air }} \mathrm{Uh}\right)-\nabla \cdot(\alpha \nabla h)=\frac{\partial \mathrm{p}}{\partial \mathrm{t}}
$$

where $\alpha$ is the thermal diffusivity of the fluid. The energy equations of the respective regions are coupled at the interface. In the case of a frost covered wall, a frost-specific wall function imposes the temperature and the heat flux based on the interaction with the frost model. Additionally the conservation equations for mass and momentum in the fluid region are taken into account. Turbulent models in the fluid region can be considered by an effective thermal diffusivity and an effective viscosity.

\subsection{Mathematical description of the frost layer}

The proposed model describes the transient defrost process of a frost layer. It is integrated into a wall function and a one-dimensional approximation of the frost layer is applied, which considers heat conduction and melting in normal direction to the wall. The reduction to a one-dimensional model is justified by the small thickness of the frost layer compared to its lateral expansion. Both wall-side and air-side defrosting can be taken into account.

The frost layer is assumed to be a porous structure consisting of areas of ice and air gaps [10]. Depending on the separate stages of the defrost process (preheating, incipient melting and appearance of an air gap) the one-dimensional transient heat conduction equation is solved for each discretized element $i$

$$
\frac{\partial \mathrm{T}_{\mathrm{i}}}{\partial \mathrm{t}}=\frac{\lambda_{\mathrm{i}}}{\mathrm{c}_{\mathrm{p}, \mathrm{i}} \rho_{\mathrm{i}}}\left(\frac{\partial^{2} \mathrm{~T}_{\mathrm{i}}}{\partial \mathrm{x}^{2}}\right)
$$

The thermal properties correspond to the three different regions of water, frost and air. During the defrost process, the initial frost thickness $\delta_{\mathrm{f}, \mathrm{t}=0}$ decreases until it falls below the stability limit, whereupon the remaining frost is assumed to drop down. The lateral displacement of the frost layer boundaries $\mathrm{s}_{\mathrm{f}}$ due to the melting process is defined by

$$
\frac{\partial \mathrm{s}_{\mathrm{f}}}{\partial \mathrm{t}}=-\frac{1}{\mathrm{~h}_{\text {melt }} \rho_{\mathrm{f}}}\left(\left.\dot{\mathrm{q}}\right|_{\mathrm{x}=\mathrm{s}_{\mathrm{L}}}+\left.\dot{\mathrm{q}}\right|_{\mathrm{x}=\mathrm{s}_{\mathrm{R}}}\right)
$$

The quantities $\left.\dot{\mathrm{q}}\right|_{\mathrm{x}=\mathrm{s}_{\mathrm{L}}}$ and $\left.\dot{\mathrm{q}}\right|_{\mathrm{x}=\mathrm{s}_{\mathrm{R}}}$ denote the heat flux across the melting interface. The thermal properties of the porous frost layer are determined based on the porosity $\varepsilon$ by averaging the properties of air and ice according to Le Gall [11]. With the assumption of 
a negligible contribution of the ambient air [12] the frost density yields $\rho_{\mathrm{f}} \approx(1-\varepsilon) \rho_{\text {ice }}$. The thermal conductivity $\lambda_{\mathrm{f}}^{\text {eff }}$ in the frost is based on the empirical correlation suggested by O’Neal [13]:

$$
\lambda_{\mathrm{f}}^{\text {eff }}=1.202 \cdot 10^{-3} \rho_{\mathrm{f}}^{0.963}
$$

A more detailed description of the model is provided in part I [14]. The boundaries of the frost model and the neighbouring regions (solid and fluid) are coupled by temperature and heat flux (temperature gradient). As an input parameter in the frost model either the temperature or the heat flux can be imposed. The quantity that is not handed from the CFD to the defrost model is returned to the CFD model. At the solid-frost interface, the temperature of the solid is imposed in the frost model, while the heat flux results. Contrary, the heat flux is enforced at the air-frost interface in order to avoid oscillations, which arise due to a high difference in the thermal diffusivity.

\section{Numerical calculations}

For the numerical simulations the finite volume code package OpenFOAM is employed. Its multi-region solver chtMultiRegionFoam uses a partitioned approach, which is applied to solve the governing equations in the solid and fluid region. The energy equations of the respective regions are coupled at the boundary interface through the present wall function. A first order implicit (bounded) Euler method has been used for the time-stepping treatment. For the velocity-pressure coupling the PIMPLE algorithm has been applied.

The calculation of the defrost process is conducted for each time step and each boundary cell. This yields an updated value for the heat flux at the solid interface and an updated value for the temperature at the gas interface. For the numerical calculations the one-dimensional frost layer of thickness $\delta_{\mathrm{f}}$ is spatially discretized into $\mathrm{N}$ elements. The governing equations for the different stages during defrosting, together with the thermophysical properties, define a system of ordinary differential equations. This system with the respective boundary conditions at the two interfaces is solved using a solver based on the Rosenbrock method (rodas23 as described in [15]). The mesh and the fluid properties in the melting zone are adapted based on the displacement of the frost layer.

\section{Test case definition}

The developed defrost model is simulated for a three-dimensional defrost process on a simplified fin geometry. The geometry is shown in Fig. 2 and consists of a plain fin with a height of $\mathrm{H}$ and width of $2 \mathrm{~B}$. 


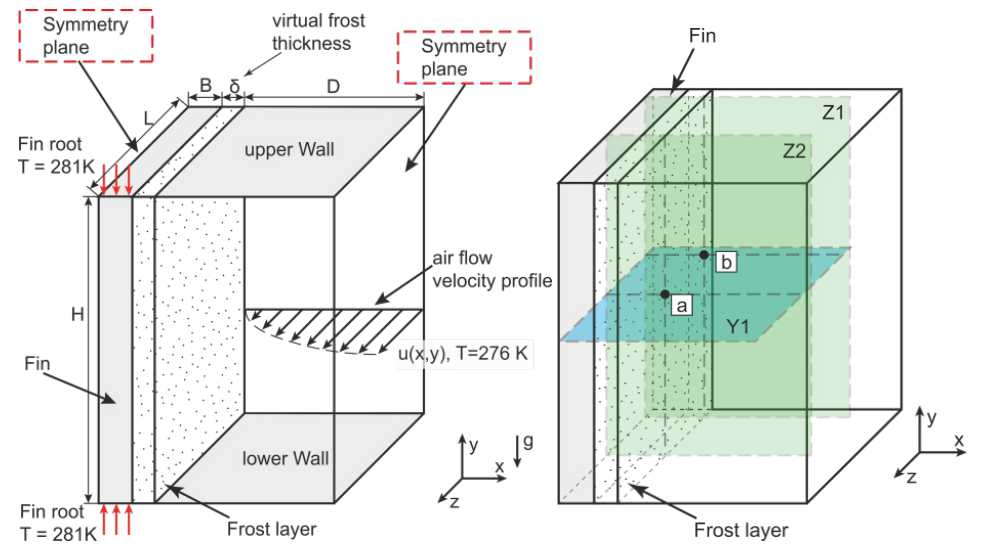

Fig. 3. Geometry of the test case (left) and position of the evaluation planes and points (right).

The fin, together with the upper and lower wall confine a flow channel of width $2 \mathrm{D}$. Due to symmetry reasons, the fin and half of the channel define the numerical domain. The no-slip boundary condition is imposed at the channel walls and at the frost surface. The fin has an initial temperature of $270 \mathrm{~K}$ and the other two channel walls are adiabatic.

Laminar air flow enters the channel in z-direction $\mathrm{Pr}=0.7$ and a parabolic velocity profile (superpositioned in $\mathrm{x}$ - and $\mathrm{y}$ - direction) is imposed at the inlet. Based on the hydraulic diameter of the rectangular channel, the Reynolds number of the flow is $\operatorname{Re} \approx 60$.

The fin is covered by a frost layer with a thickness of $\delta_{\mathrm{F}}$ and a porosity of $\varepsilon$. The porosity is assumed to be constant over the defrost process (no densification). The model assumes a maximum water film layer of $\delta_{\mathrm{W}, \max }$ and a minimal frost layer thickness (stability limit) of $\delta_{\mathrm{F}, \text { min. }}$. The values are based on prior works of [5] and presented in Tab. 1.

Table 1. Input parameters for the three-dimensional simulation.

\begin{tabular}{|c|c|c|c|}
\hline Parameter & & Unit & Value \\
\hline Fin height & $\mathrm{H}$ & $\mathrm{mm}$ & 14 \\
\hline Fin length & $\mathrm{L}$ & $\mathrm{mm}$ & 8 \\
\hline Fin width & $\mathrm{B}$ & $\mathrm{mm}$ & 1.5 \\
\hline Channel width & $\mathrm{D}$ & $\mathrm{mm}$ & 10 \\
\hline Average air inlet velocity & $\mathrm{U}$ & $\mathrm{m} / \mathrm{s}$ & 0.05 \\
\hline Fin root temperature & $\mathrm{T}_{\text {Wall }}$ & $\mathrm{K}$ & 281 \\
\hline Air inlet temperature & $\mathrm{T}_{\text {Air }}$ & $\mathrm{K}$ & 276 \\
\hline Initial frost temperature & $\mathrm{T}_{\mathrm{F}}$ & $\mathrm{K}$ & 270 \\
\hline Initial frost thickness & $\delta_{\mathrm{F}}$ & $\mathrm{mm}$ & 1 \\
\hline Minimal frost layer thickness & $\delta_{\mathrm{F}, \min }$ & $\mathrm{mm}$ & 0.2 \\
\hline Maximal water layer thickness & $\delta_{\mathrm{W}, \mathrm{max}}$ & $\mathrm{mm}$ & 0.2 \\
\hline Frost porosity & $\varepsilon$ & - & 0.5 \\
\hline Mesh size (3D-model) & $\Delta \mathrm{x}, \Delta \mathrm{y}, \Delta \mathrm{z}$ & $\mathrm{mm} /$ cell & $0.125(0.25)$ \\
\hline Mesh size frost & $\Delta \mathrm{xf}$ & $\mathrm{mm} /$ cell & $0.01(0.02)$ \\
\hline Time step size & $\Delta \mathrm{t}$ & $\mathrm{s}$ & 0.001 \\
\hline \multicolumn{3}{|c}{} \\
\hline
\end{tabular}

\section{Results}

In the following section, numerical simulation results of the test case are presented for a set of cross-sections and positions at different instances of time. Figure 4 illustrates the 
location of the sampling planes $(Z 1, Z 2)$ and points $(a-b)$. The end time of the simulation is 200s. After $150 \mathrm{~s}$, the frost layer thickness falls below the stability threshold at any position of the wall. Thus, according to the defrost model, the wall is covered by a remaining water layer.

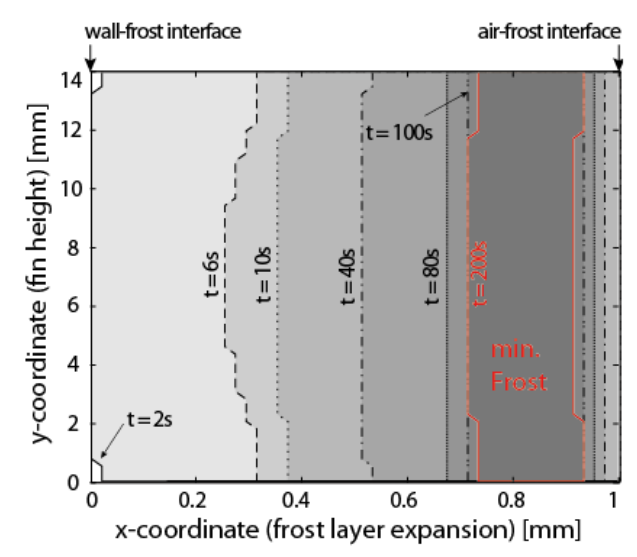

(a) Cross section $\mathrm{Z1}$ according to Fig. 4

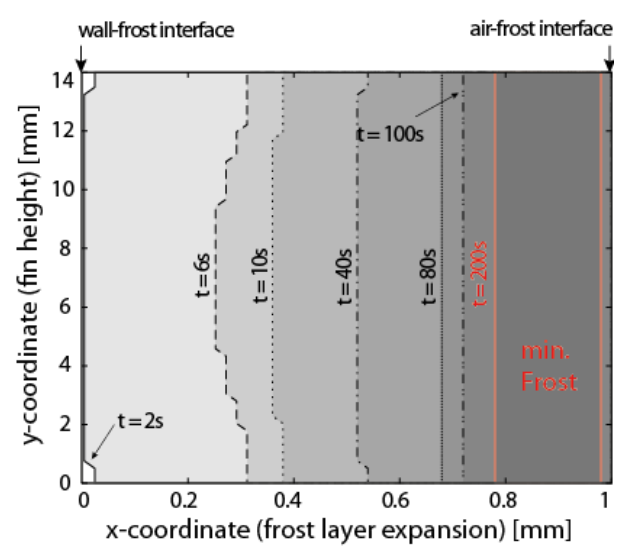

(b) Cross section Z2 according to Fig. 4

Fig. 5. Melting process of the frost borders for two different streamwise locations. For both cross sections wall side heating is dominant, while air-side melting mainly occurs at the beginning of the channel. Cross sections Z1 (left) and Z2 (right) according Fig. 6.

Figure 3 presents the defrost process for two planes perpendicular to the air flow (Z1 and Z2). Due to the higher heat transfer rate from fin to frost, the wall-side melting process is significantly stronger than the air-side one. As a consequence, a large gap (filled with water and air) between the fin and the remaining frost layer develops, acting as an additional thermal resistance, slowing down the defrost process. Five seconds after the defrost process has started, the frost layer thickness has decreased by at least $0.2 \mathrm{~mm}$, which corresponds to the maximal thickness of the water layer. The heat input on the top and the bottom side of the fin causes an inhomogeneous melting rate, with a minimum in the fin centre $(y=7 \mathrm{~mm})$. During the defrost process, the melting rate becomes more homogeneous over the fin height, since the thermal resistance of the air gap dominates the process (the thermal resistance in the fin becomes negligible). Differences in the air-side melting process are shown by a comparison of the cross sections Z1 and Z2. While no significant air-side melting appears at cross section $\mathrm{Z2}$, the frost layer decreases by approximately $0.1 \mathrm{~mm}$ close to the air inlet (Z1). The temporal development of the frost layer at the two sampling positions (a-b) is presented in Fig. 7. 


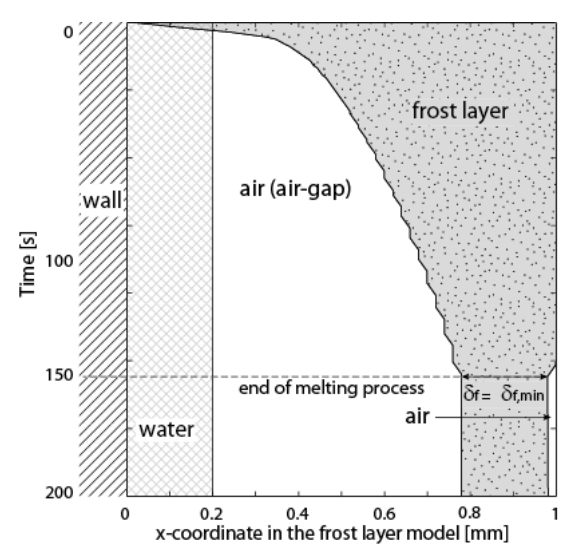

(a) Probe location a according to Fig. 2

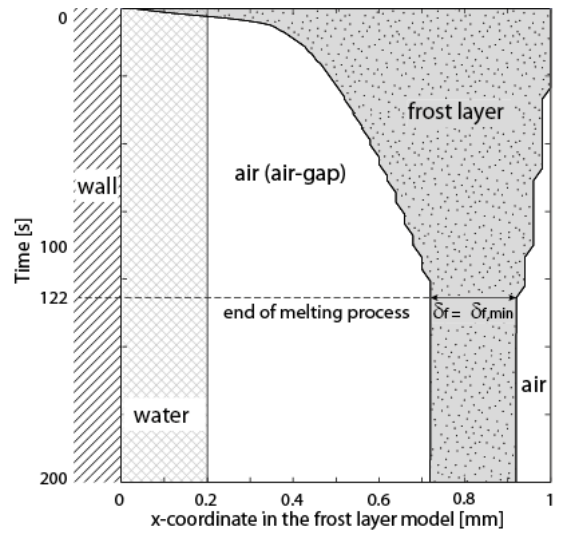

(a) Probe location $\mathbf{b}$ according to Fig. 2

Fig. 7. Temporal development of the frost layer at two distinct locations a (left) and b (right).

The three different regions (water, air, and frost) are indicated by different patterns and the time increases from top to bottom in each plot. For both sampling positions, the wall-side melting process is very similar up to the point in time when $\delta=\delta_{\mathrm{F} \text {,min. }}$. In the beginning of the process, the water layer develops, reaching its maximum thickness at $t=$ $3.5 \mathrm{~s}$. With the increasing thickness of the air gap and the increasing thermal resistance, the fin-side melting process slows down, whereas the air-side melting process (apparent at position a) reveals a linear behaviour due to the constant flow rate. The temperature distributions for fin, frost, and air regions are presented in Fig. 8 at two different locations $(\mathrm{Z} 1$ and $\mathrm{Z2})$ and two different instances of time $\mathrm{t}=6 \mathrm{~s}$ and $\mathrm{t}=100 \mathrm{~s}$.

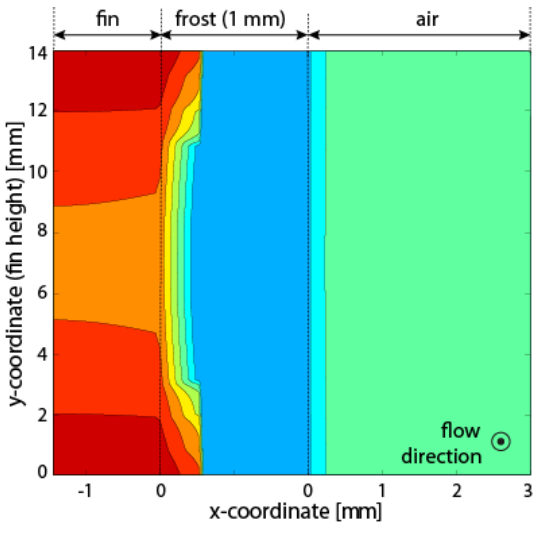

(a) Section close to the air inlet, $\mathrm{t}=6 \mathrm{~s}$.

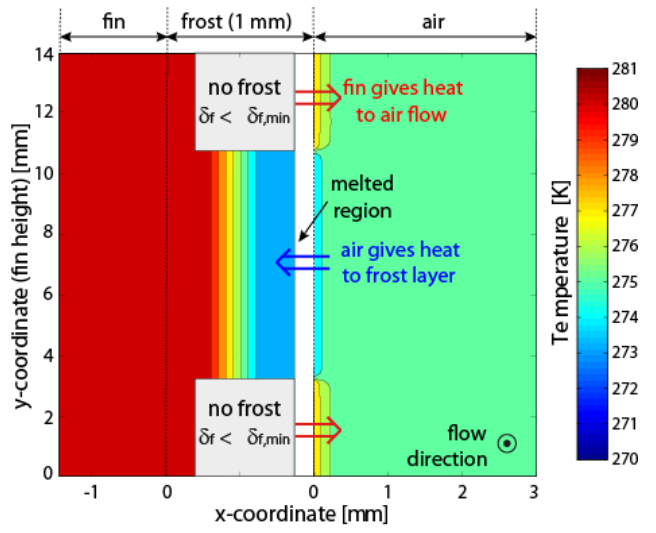

(b) Section close to the air inlet, $\mathrm{t}=100 \mathrm{~s}$.

Fig. 8. Temperature field for a section close to the air inlet at $t=6 \mathrm{~s}$ and $\mathrm{t}=100 \mathrm{~s}$.

The temperature field close to the air inlet at $t=6 \mathrm{~s}$ shows a strong temperature gradient over the fin height. This gradient is caused by the heating of the fin root and the transient boundary condition within the first five seconds. In addition, a smaller temperature gradient is apparent over the fin width, resulting from the heat transfer to the frost layer. The temperature distribution in the frost is characterized by two different regions. In the center region $(3<\mathrm{y}<11 \mathrm{~mm})$, the water layer between the frost and the wall exhibits a linear temperature gradient. Close to the fin roots, the maximum water layer thickness is exceeded 
such that an additional air gap develops. Due to the different thermal conductivity of air and water, a strong temperature gradient in the air gap establishes. The remaining frost layer is characterized by an almost homogeneous temperature, just below melting temperature. Note that there are no significant variations of the temperature distribution in the fin and the frost with respect to the flow direction (z-coordinate) at $t=6 \mathrm{~s}$.

For $\mathrm{t}=100 \mathrm{~s}$, the temperature in the entire fin and the covering water layer is similar to the temperature of the fin root in both sections. The dominating thermal resistance of the air gap limits the heat transfer from the water layer to the frost. In Fig. 8, two different regions exist. Close to the fin roots, the frost layer thickness falls below the stability limit. Thus, the remaining frost layer drops down and a water layer remains. As a consequence, the air temperature increases due to a wall-side heating above the air inlet temperature. In the center region, the defrost process is ongoing with a heat flux from the ambient air to the frost. The white area illustrates the amount of frost melted by air-side defrosting. In the center section of the channel (not shown), the increased temperature boundary layer reduces the air-side defrosting. Over the entire height of the fin, the frost layer thickness has not reached the stability limit yet.

\section{Conclusion}

In this study, a one-dimensional defrost model developed in [14] has been included in a multi-region three-dimensional finite volume code through a wall-function. The wallfunction couples the temperatures and the heat transfer rates (temperature gradients) of the different regions. The defrost model itself considers the frost layer, and additional resistances due to a water layer and an air gap. Wall- and air-side heating can be applied resulting in a two-sided melting process.

Despite the fact that the presented approach does not account for the expansion of the frost layer into the air region, the wall-function based model can be used to simulate the defrost process in large and complex geometries.

A simple three-dimensional test case of a frost-covered fin is designed to identify the capabilities of the model. Results of first numerical simulations show local variations of the defrost process due to the temperature distribution along the height of the fin and a developing boundary layer in the air flow.

\section{References}

1. C. Machielsen, H. Kerschbaumer, Int. J. Refrig. 12, 5 (1989)

2. A. Alebrahim, S. Sherif, Proceedings of the Institution of Mechanical Engineers, Part

C: Journal of Mechanical Engineering Science 216, 6 (2002)

3. C. T. Sanders, Ph.D. thesis, University of Delft (1974)

4. N. Hoffenbecker, S. Klein, D. Reindl, Int. J. Refrig. 28, 4 (2005)

5. J.A. Dopazo, J. Fernandez-Seara, F.J. Uhia, R. Diz, Int. J. Refrig 33, 4 (2010)

6. S. Ellgas, M. Pfitzner, M. Numer, Heat Tr. A-Appl. 53, 5 (2007)

7. J.-S. Kim, K.-S. Lee, S.-J. Yook, Int. J. Heat Mass Tran. 52, 11-12 (2009)

8. K. Lenic, A. Trp, B. Frankovic, Int. J. Heat Mass Tran. 52, (2008)

9. C. Hirt, B. Nichols, J. Comput. Phys. 39, 1 (1981)

10. Y.-X. Tao, R.W. Besant, K.S. Rezkallah, Int. J. Heat Mass Tran. 36, (1993)

11. R. Le Gall, J. Grillot, Int. J. Heat Mass Tran 40, (1997)

12. K. Prölss, G. Schmitz, The Modelica Association 1, (2006) 
13. D. O'Neal, D. Tree, ASHRAE Trans. 91 (1985)

14. S.H. van Buren, E. Hertle, P. Figueiredo, R. Kneer, W. Rohlfs (2017)

15. A. Sandu, J. Verwer, J. Blom, E. Spee, G. Carmichael, Atmos. Environ. 31 (1997) 